\title{
Small Boat Detection Using OFDM Radar
}

\author{
Uday Kumar SINGH ${ }^{1}$, Vimal BHATIA ${ }^{1}$, Amit Kumar MISHRA ${ }^{2}$ \\ ${ }^{1}$ Dept. of Electrical Engineering, Indian Institute of Technology Indore, India \\ ${ }^{2}$ Dept. of Electrical Engineering, University of Cape Town, South Africa
}

\{phd1501202001,vbhatia\}@iiti.ac.in, amit.mishra@uct.ac.za

Submitted December 31, 2018 / Accepted September 25, 2019

\begin{abstract}
Orthogonal frequency division multiplexing (OFDM) based radar systems have recently attracted a lot of research interest. However, demonstration of OFDM radar's capability for target detection using real data is not reported in the open literature. In this work, we demonstrate a method to employ OFDM radar for small boat detection. For this objective, we propose a technique to generate radar return for OFDM waveform using collected radar return data when stepped frequency waveform is transmitted. We, then, derive system model for the estimated radar return data specific to OFDM waveform. Further, a detection test is proposed for the derived signal model and surveillance environment. Close match between the derived analytical expressions and simulation results validates the proposed detector's performance.
\end{abstract}

\section{Keywords}

OFDM, stepped frequency, radar return, small boat, sea clutter

\section{Introduction}

Detection of a small boat using radar is of significant importance for security and surveillance. Waves produced by the motion of a boat introduce interference of high spikes which cause degradation in the performance of conventional radar systems [1], [2]. Sea waves cause sea clutter which makes detection of small targets in an ocean difficult [3], [4]. Typically, sea clutter follows a non-Gaussian distribution [3], which affects detection and tracking of a small boat in the sea. For small boat detection in a high clutter environment, many algorithms [3-9] have been proposed in the literature. Some algorithms have been validated by using real sea environment (measurement) data. For instance, in [2], the adaptive linear quadratic detector was used for the detection of small Rigid-hulled inflatable boat (RIB) from real data that has been recorded with a medium resolution X-band radar. In [5], a detection algorithm with constant false alarm rate is derived, with performance analysis over both the simulated and the real sea data. However, validation of previously proposed algorithms is done using conventional pulsed radar waveforms. Moreover, the high amplitude of side-lobes in the ambiguity plot of pulsed waveform [5] results in false alarms and hence worsens target detection probability. Hence, there is an opportunity to explore the performance of other types of waveforms which can improve the detection performance of maritime radar systems.

One of the newer types of waveforms explored for radar is a multi-carrier waveform. Studies have shown potential merits of multi-carrier waveform like orthogonal frequency division multiplexing (OFDM) over traditionally used waveforms [10-18]. Some of these advantages includes waveform diversity and lower side lobe levels in ambiguityfunction [19-21]. However, to the best of authors' knowledge, the merits of OFDM waveform have not yet been verified and validated using real radar return from sea clutter. The system model considered in the literature either considers a noise-free environment or the environment where clutter and thermal noise follows a Gaussian distribution. This makes the existing algorithms unsuitable for practical radar environment, which is affected by the interference that follows a non-Gaussian distribution. Moreover, as [22], [23], and [24] lack in measuring the performance of target detector, their robustness and suitability against dynamic interference environment is not guaranteed.

Major contributions of this work are as follows: We propose a technique for the detection of small boat by utilizing an estimated radar return for the OFDM waveform. The estimation of radar return is done in two steps. In the first step, impulse response (IR) of the radar system for a single coherent pulse interval (CPI) is estimated by the method of least squares (LS) [25]. Then, the estimated IR is used for the estimation of OFDM radar return. Further, a detailed analytical expression for the system model corresponding to the estimated data at a particular range gate is proposed. Furthermore, the generalized likelihood ratio test (GLRT) based sub-optimum detector is proposed, and its performance is compared with the existing normalized matched filter (NMF) (optimum for conventional radar) [26]. Finally, to verify improvement in the performance of detection test, the analytical expression of the probability of false alarm $\left(P_{\mathrm{FA}}\right)$, and the probability of detection $\left(P_{\mathrm{D}}\right)$ for the proposed detection test are derived. 
Rest of the paper is organized as follows: In Sec. 2, method to transform radar return data for stepped frequency (SF) waveform into radar return for OFDM waveform is described. Section 3 describes the proposed OFDM radar system model for the considered surveillance environment. Section 4, discusses the proposed modified target detection algorithm for a single range gate and fixed CPI. Further, in Subsec. 4.3, analytical expression for $P_{\mathrm{D}}$ and $P_{\mathrm{FA}}$ for the proposed detection algorithm under Gaussian assumption are derived. In Sec. 5, simulation results for the estimated IR of the radar channel and the proposed detector's performance obtained from estimated OFDM radar return data are given. Performance of the proposed detector obtained by the analytical expressions for receiver operating characteristic (ROC) and $P_{\mathrm{FA}}$ is discussed next. Finally, Section 6 concludes the work and also presents possible future directions.

Notation: Scalar variables (constants) are denoted by lower (upper) case letters. Vectors (matrices) are denoted by boldface lower (upper) case letters. Superscripts $(\cdot)^{\mathrm{T}},(\cdot)^{\mathrm{H}}$ denote transpose, and complex conjugate transpose, respectively. $\mathbb{E}[\cdot]$ denotes statistical expectation operation. $\mathbb{C}$ and $\mathbb{R}$ denotes the set of complex and real numbers, respectively. diag, tr and $\mathcal{R}$ are respectively the diagonal, trace, and rank operation over a matrix.

\section{Estimation of Radar Return}

In this section, we describe the procedure used for the estimation of radar return data set for OFDM waveform.

To obtain the radar returns for OFDM waveform, we model the complete radar system (including radar channel, down converter, a pulse compressor, and sampler) which was used to record the original radar returns as finite impulse response (FIR) filter. Two main steps of data transformation are an estimation of radar system IR and estimation of the response of the radar system for OFDM waveform (considered as echoes for OFDM waveform).

\subsection{Estimation of Radar Impulse Response}

Without loss of generality, let the radar system IR be approximated as an complex FIR filter IR with $K$-unknown complex coefficients $\mathbf{h} \in \mathbb{C}^{K \times 1}=[h(0), h(1), \cdots, h(K-1)]^{\mathrm{T}}$. Following this assumption, the response of an unknown radar system in terms of the transmitted signal in the time domain is given by

$$
y_{\mathrm{SFW}}(n)=\sum_{k=0}^{K-1} h(k) x_{\mathrm{SFW}}(n-k)+e(n), n=0,1, \ldots, N-1
$$

where $e(n)$ is the error in approximating the radar system as FIR filter [27] , [25], $n=(0,1, \ldots, N-1)$ represents the index for the dimension of the fast time within one CPI, $y_{\mathrm{SFw}}(n)$ represents the radar return for SF waveform.
For known values of $y_{\mathrm{SFW}}(n)$ and $x_{\mathrm{SFW}}(n)$, values of filter coefficients $\mathbf{h}$ can be estimated by minimizing the LS cost function [25], given as

$$
\begin{aligned}
J(\mathbf{h}) & =\sum_{n=0}^{N-1}|e(n)|^{2} \\
& =\|\mathbf{e}\|^{2}
\end{aligned}
$$

where, $\mathbf{e} \in \mathbb{C}^{N \times 1}=[e(0), e(1), \cdots, e(N-1)]^{\mathrm{T}}$.

Term $\mathbf{e}$ in (2), can then be written as

$$
\mathbf{e}=\mathbf{y}_{\mathrm{SFW}}-\mathbf{X}_{\mathrm{SFW}} \mathbf{h}
$$

where $\mathbf{y}_{\mathrm{SFW}} \in \mathbb{C}^{N \times 1}=\left[y_{\mathrm{SFW}}(0), y_{\mathrm{SFW}}(1), \cdots, y_{\mathrm{SFW}}(N-1)\right]^{\mathrm{T}}$,

$$
\begin{aligned}
& \mathbf{X}_{\mathrm{SFW}} \in \mathbb{C}^{N \times K}= \\
& {\left[\begin{array}{cccc}
x_{\mathrm{SFW}}(0) & x_{\mathrm{SFW}}(-1) & \ldots & x_{\mathrm{SFW}}(-(K-1)) \\
x_{\mathrm{SFW}}(1) & x_{\mathrm{SFW}}(0) & \ldots & x_{\mathrm{SFW}}(1-(K-1)) \\
\vdots & \vdots & \vdots & \vdots \\
x_{\mathrm{SFW}}(N-1) & x_{\mathrm{SFW}}(N-2) & \ldots & x_{\mathrm{SFW}}(N-(K-1))
\end{array}\right]}
\end{aligned}
$$

and $\mathbf{h}=[h(0), h(1), \cdots, h(K-1)]^{\mathrm{T}}$.

After substituting (3) into (2), we get

$$
\begin{aligned}
J(\mathbf{h}) & =\left\|\mathbf{y}_{\mathrm{SFW}}-\mathbf{X}_{\mathrm{SFW}} \mathbf{h}\right\|^{2} \\
& =\left(\mathbf{y}_{\mathrm{SFW}}-\mathbf{X}_{\mathrm{SFW}} \mathbf{h}\right)^{\mathrm{H}}\left(\mathbf{y}_{\mathrm{SFW}}-\mathbf{X}_{\mathrm{SFW}} \mathbf{h}\right) \\
& =\mathbf{y}_{\mathrm{SFW}}^{\mathrm{H}} \mathbf{y}_{\mathrm{SFW}}-\mathbf{y}_{\mathrm{SFW}}^{\mathrm{H}} \mathbf{X}_{\mathrm{SFW}} \mathbf{h}-\left(\mathbf{X}_{\mathrm{SFW}} \mathbf{h}\right)^{\mathrm{H}} \mathbf{y}_{\mathrm{SFW}} \\
& +\left(\mathbf{X}_{\mathrm{SFW}} \mathbf{h}\right)^{\mathrm{H}}\left(\mathbf{X}_{\mathrm{SFW}} \mathbf{h}\right) .
\end{aligned}
$$

To obtain the value of $\mathbf{h}$ which minimizes (4), the differentiation of (4) with respect to $\mathbf{h}$ is equated to zero as

$$
\mathbf{X}_{\mathrm{SFW}}{ }^{\mathrm{H}} \mathbf{X}_{\mathrm{SFW}} \mathbf{h}-\mathbf{X}_{\mathrm{SFW}}^{\mathrm{H}} \mathbf{y}_{\mathrm{SFW}}=0 .
$$

From (5), the estimate of $\mathbf{h}$ is given by

$$
\hat{\mathbf{h}}=\left(\mathbf{X}_{\mathrm{SFW}}^{\mathrm{H}} \mathbf{X}_{\mathrm{SFW}}\right)^{-1} \mathbf{X}_{\mathrm{SFW}}^{\mathrm{H}} \mathbf{y}_{\mathrm{SFW}} \cdot
$$

\subsection{Estimation of Radar Response for OFDM Pulsed Waveform}

After estimating the filter coefficients of the radar system, we find the response of the radar system (modeled by the FIR system) for the OFDM waveform. For IR estimation and radar return data estimation, the response of the radar system is calculated for single CPI, and given by the convolution of $\hat{h}(k)$ and $x_{\mathrm{OFDM}}(k)$. The following relationship in time domain describes the response of the radar system for OFDM waveform

$$
y_{\mathrm{OFDM}}(n)=\sum_{k=0}^{K-1} \hat{h}(k) x_{\mathrm{OFDM}}(n-k), n=0,1, \ldots, N-1 .
$$




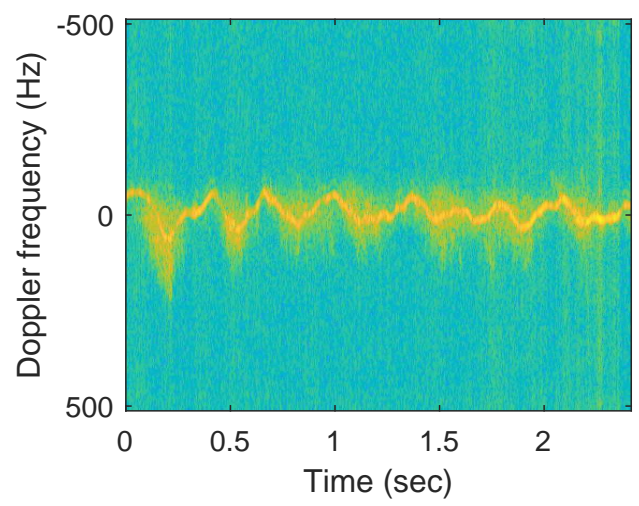

(a) Doppler spectrum of measured OFDM radar return.

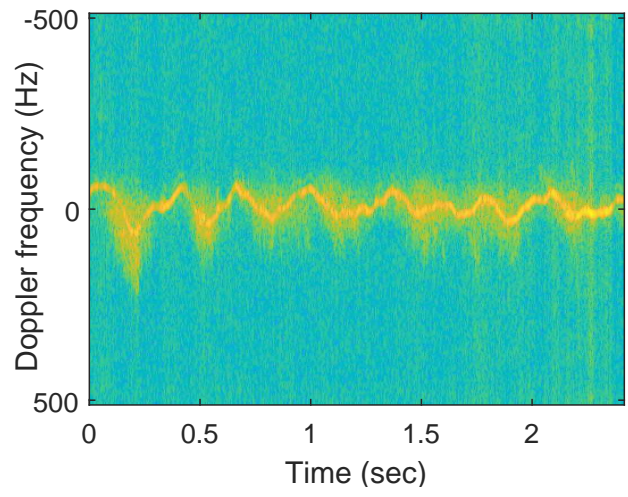

(b) Doppler spectrum of original SF radar return.

Fig. 1. Comparative Doppler spectrum of measured OFDM radar return and original SF radar return.

Arranging $y_{\text {OFDM }}(n)$ in vector yields the estimated radar return for OFDM waveform as

$$
\mathbf{y}_{\text {OFDM }}=\mathbf{X}_{\text {OFDM }} \hat{\mathbf{h}} \text {. }
$$

Further, Doppler processing over measured OFDM radar return and available SF radar return are shown in Fig. 1a and Fig. 1b, respectively. As shown in Fig. 1a and Fig. 1b, the Doppler spectrum of measured OFDM radar return follows the Doppler spectrum of original SF radar return. This validates the correctness of the measured OFDM radar return.

\section{Proposed System Model}

In this section, a system model for the estimated data is proposed, and the analytical expression corresponding to the estimated scattered radar return for the OFDM waveform is described.

We consider an OFDM waveform $s(t)$ of $L$-subcarriers modulated by complex $a_{l}$ phase codes from the set $\mathbf{a}=\left[a_{0}, a_{1}, \ldots, a_{L-1}\right]$. If the sub-carriers in frequency domain are spaced by $\Delta f$, then the expression for $s(t)$ is given by

$$
s(t)=\sum_{l=0}^{L-1} a_{l} \exp (\mathrm{j} 2 \pi l \Delta f t) \text { for } 0 \leq t \leq T_{\mathrm{o}}
$$

where $T_{\mathrm{o}}$ is the OFDM waveform duration without cyclic prefix. The sub-carriers are orthogonal for $T_{\mathrm{o}}=\frac{1}{\Delta f}$. In this work, the echoes of the prior pulse reach the receiver before the next pulse is transmitted, thereby avoiding inter-symbol interference [28].

Let $f_{\mathrm{c}}$ be the center frequency of transmission; then the transmitted signal is given by

$$
\tilde{S}(t)=s(t) \exp \left(\mathrm{j} 2 \pi f_{\mathrm{c}} t\right)=\sum_{l=0}^{L-1} a_{l} \exp \left(\mathrm{j} 2 \pi f_{l} t\right)
$$

where $f_{l}=f_{\mathrm{c}}+l \Delta f$ is the sub-carrier frequency.
Radar return corresponding to $\tilde{S}(t)$ is the sum of delayed and time scaled version of $\tilde{S}(t)$. Let us consider that the radar surveillance environment consists of $P$ scatterers out of which one is the target and others represent clutter. Scatterers are at distances $\left(R_{p}\right)_{p=1}^{P}$, moving with a velocity vector $\left(\overrightarrow{\boldsymbol{v}}_{p}\right)_{p=1}^{P}$ and causing the delay $\left(\tau_{p}\right)_{p=1}^{P}$. The complex scattering coefficient $\left(x_{l p}\right)$ of $p^{\text {th }}$ scatterer for $l^{\text {th }}$ sub-carrier is unknown but deterministic.

After making these basic assumptions, the received radar return for the $l^{\text {th }}$ sub-carrier and for the $p^{\text {th }}$ scatterer is given by

$$
\tilde{r}_{l p}(t)=x_{l p} \tilde{s}_{l}\left(\gamma_{p}\left(t-\tau_{p}\right)\right)+\tilde{w}_{l}(t)
$$

where $\tilde{s}_{l}=a_{l} \exp \left(\mathrm{j} 2 \pi f_{l} t\right), \gamma_{p}=1+\beta_{p}$ where $\beta_{p}=\frac{2\left\langle\overrightarrow{\boldsymbol{v}}_{p}, \mathbf{u}\right\rangle}{c}$ is the relative Doppler shift of the $p^{\text {th }}$ scatterer, $c$ is the velocity of light, and $\tilde{w}_{l}$ represents the thermal noise along the $l^{\text {th }}$ subchannel. Hence, the received signal return from $P$ scatterer along $L$ subchannels is given by

$$
\begin{aligned}
\tilde{r}(t)= & \sum_{p=1}^{P} \sum_{l=0}^{L-1} r_{l p}(t) \\
= & \sum_{p=1}^{P} \sum_{l=0}^{L-1} x_{l p} \tilde{s}_{l}\left(\gamma_{p}\left(t-\tau_{p}\right)\right)+\tilde{w}(t) \\
= & \sum_{p=1}^{P} \sum_{l=0}^{L-1} a_{l} x_{l p} \exp \left(\mathrm{j} 2 \pi f_{l} \gamma_{p}\left(t-\tau_{p}\right)\right)+\tilde{w}(t) \\
= & \sum_{p=1}^{P} \sum_{l=0}^{L-1} a_{l} x_{l p} \exp \left(\mathrm{j} 2 \pi f_{l}\left(1+\beta_{p}\right)\left(t-\tau_{p}\right)\right)+\tilde{w}(t) \\
= & \sum_{p=1}^{P} \sum_{l=0}^{L-1} a_{l} x_{l p} \exp \left(\mathrm{j} 2 \pi f_{l}\left(t-\tau_{p}\right)\right) \exp \left(\mathrm{j} 2 \pi f_{l} \beta_{p}\left(t-\tau_{p}\right)\right) \\
+ & \tilde{w}(t) \\
= & \left\{\sum_{p=1}^{P} \sum_{l=0}^{L-1} a_{l} x_{l p} \exp \left(\mathrm{j} 2 \pi l \Delta f\left(t-\tau_{p}\right)\right)\right. \\
& \left.\times \exp \left(\mathrm{j} 2 \pi f_{l} \beta_{p}\left(t-\tau_{p}\right)\right)\right\} \exp \left(\mathrm{j} 2 \pi f_{\mathrm{c}} t\right)+\tilde{w}(t)
\end{aligned}
$$


Thus, the corresponding complex envelope after removing the carrier $\left(\exp \left(\mathrm{j} 2 \pi f_{\mathrm{c}} t\right)\right)$ is given by

$$
\begin{aligned}
r(t)= & \sum_{p=1}^{P} \sum_{l=0}^{L-1} a_{l} x_{l p} \exp \left(\mathrm{j} 2 \pi l \Delta f\left(t-\tau_{p}\right)\right) \\
& \exp \left(\mathrm{j} 2 \pi f_{l} \beta_{p}\left(t-\tau_{p}\right)\right)+w(t) .
\end{aligned}
$$

Since the estimated data set is the radar return from the single target, (11) can be simplified by separating the terms for the phase shifts corresponding to the target. Remaining $P-1$ terms in the outer summation corresponds to the sea clutter. Thus, (11) can be written as

$$
\begin{aligned}
r(t)= & \sum_{l=0}^{L-1} a_{l} x_{l t} \exp \left(\mathrm{j} 2 \pi l \Delta f\left(t-\tau_{t}\right)\right) \\
& \times \exp \left(\mathrm{j} 2 \pi f_{l} \beta_{t}\left(t-\tau_{t}\right)\right)+c(t)+w(t)
\end{aligned}
$$

where $x_{l t}, \tau_{t}$, and $\beta_{t}$ are the scattering coefficient, delay and relative Doppler shift respectively, corresponding to the target, and $c(t)$ represent the clutter.

Before further processing, (12) is sampled with the sampling interval of $m T_{\mathrm{PRI}}+\tau_{t}, m=0,1, \ldots, M-1$, where $m$ is the index for slow time dimension, $T_{\mathrm{PRI}}$ is a sampling interval across slow time which is equal to the PRI of an OFDM signal and $M$ is the number of temporal measurements within a given CPI. Hence, the discrete complex envelope of the received signal at the output of the $l^{\text {th }}$ subchannel is

$$
\begin{array}{r}
r_{l}(m)=a_{l} x_{l_{t}} \exp \left(\mathrm{j} 2 \pi m f_{l \mathrm{D}_{\mathrm{t}}} T_{\mathrm{PRI}}\right)+c_{l}(m)+w_{l}(m), \\
l=0,1, \ldots, L-1, m=0,1, \ldots, M-1
\end{array}
$$

where the constant $\exp \left(\mathrm{j} 2 \pi m l \Delta f T_{\mathrm{PRI}}\right)$ is considered along with the scattering coefficient $\left(x_{l t}\right)$, and $f_{l \mathrm{D}_{\mathrm{t}}}=\left(f_{\mathrm{c}}+l \Delta f\right) \frac{2\left\langle\overrightarrow{\boldsymbol{v}}_{p_{t}}, \mathbf{u}\right\rangle}{c}$ is the Doppler shift along $l^{\text {th }}$ subchannel.

Arranging returns of all $L$ subchannels into one $L \times 1$ dimension vector, we get

$$
\mathbf{r}(m)=\mathbf{A} \mathbf{X}_{t} \boldsymbol{\phi}(m)+\mathbf{c}(m)+\mathbf{w}(m), m=0,1, \ldots, M-1
$$

where

- $\mathbf{r}(m)=\left[r_{0}(m), r_{1}(m), \ldots, r_{N-1}(m)\right]^{\mathrm{T}}$ is the $L \times 1$ dimension vector of sub-carrier return,

- $\mathbf{A}=\operatorname{diag}\left[a_{0}, a_{1}, \ldots, a_{L-1}\right]$ is the $L \times L$ diagonal matrix of optimized (reduced peak to average power ratio (PAPR)) transmitted phase codes,

- $\mathbf{X}_{t}=\operatorname{diag}\left[x_{0_{t}}, x_{1_{t}}, \ldots, x_{(L-1)_{t}}\right]$ is the $L \times L$ diagonal matrix of scattering coefficients across $L$ subchannels,

- $\boldsymbol{\phi}(m)=\left[\exp \left(\mathrm{j} 2 \pi m f_{0 \mathrm{D}_{\mathrm{t}}} T_{\mathrm{PRI}}\right), \exp \left(\mathrm{j} 2 \pi m f_{1 \mathrm{D}_{\mathrm{t}}} T_{\mathrm{PRI}}\right), \ldots\right.$, $\left.\exp \left(\mathrm{j} 2 \pi m f_{(L-1) \mathrm{D}_{\mathrm{t}}} T_{\mathrm{PRI}}\right)\right]^{\mathrm{T}}$ is the $L \times 1$ dimension vector of phase shifts corresponding to different Doppler frequencies $f_{\mathrm{D}_{\mathrm{t}}}=\left[f_{0 \mathrm{D}_{\mathrm{t}}}, f_{1 \mathrm{D}_{\mathrm{t}}}, \ldots, f_{(L-1) \mathrm{D}_{\mathrm{t}}}\right]$ across $L$ subchannels,
- $\mathbf{c}(m)=\left[c_{0}(m), c_{1}(m), \ldots, c_{L-1}(m)\right]^{\mathrm{T}}$ is the $L \times 1$ dimension vector of sea clutter return across $L$ subchannels,

- $\mathbf{w}(m)=\left[w_{0}(m), w_{1}(m), \ldots, w_{L-1}(m)\right]^{\mathrm{T}}$ is the $L \times 1 \mathrm{di}-$ mension vector of the samples of additive white Gaussian noise (AWGN) across $L$ subchannels.

Subsequently, concatenating all the temporal radar return data column-wise into a matrix of dimension $L \times M$, the mathematical description for the OFDM radar return matrix whose columns corresponds to the estimated OFDM radar return data is given by

$$
\mathbf{R}=\mathbf{A} \mathbf{X}_{t} \boldsymbol{\Phi}+\mathbf{C}+\mathbf{W}
$$

where

- $\mathbf{R}=[\mathbf{r}(0), \mathbf{r}(1), \ldots, \mathbf{r}(M-1)]$ is the $L \times M$ matrix of all temporal returns,

- $\boldsymbol{\Phi}=[\boldsymbol{\phi}(0), \boldsymbol{\phi}(1), \ldots, \boldsymbol{\phi}(M-1)]$ is the $L \times M$ matrix of phase shifts corresponding to temporal components $m$,

- $\mathbf{C}=[\mathbf{c}(0), \mathbf{c}(1), \ldots, \mathbf{c}(M-1)]$ is the $L \times M$ matrix representing sea clutter returns,

- $\mathbf{W}=[\mathbf{w}(0), \mathbf{w}(1), \ldots, \mathbf{w}(M-1)]$ is the $L \times M$ matrix representing AWGN samples.

\section{Target Detection Test}

In this section, we propose a target detection test for the considered OFDM radar model, described by (15). We first describe the statistical behavior of the sea clutter. For target detection, the proposed detection algorithm is described next. Following this, to verify the correctness of the detection test, an analytical expression for $P_{\mathrm{D}}$ and $P_{\mathrm{FA}}$ of the detection test statistics is derived. The obtained theoretical expression for $P_{\mathrm{D}}$ and $P_{\mathrm{FA}}$ validates the ROC of the proposed detection algorithm.

\subsection{Statistical Description of Sea Clutter}

Received radar echoes in the sea environment are affected by the interference produced by the motion of small waves. Without loss of generality, the effect of thermal noise W from (15) is ignored, since, the power spectrum of noise is usually $20 \mathrm{~dB}$ below that of the clutter [29], [30]. Statistically, the sea clutter follows a non-Gaussian distribution [3], [4]. In particular, K-distribution, which describes the spherically invariant random process (SIRP), is considered as a suitable fit for the distribution of sea clutter.

Hence, from [31], $\mathbf{c}(m) \forall m$ can be described as

$$
\mathbf{c} \in \mathbb{C}^{M \times 1}=\sqrt{\sigma} \mathbf{z}
$$

where index $m$ is dropped for simplicity and $\mathbf{z} \in \mathbb{C}^{M \times 1}$ is the speckle component of the sea clutter. The speckle component is modeled as a zero mean multivariate complex correlated 
Gaussian distributed random variable with unknown covariance matrix $\boldsymbol{\Sigma}_{\mathbf{z z}}=\mathbb{E}\left\{\mathbf{z z}^{\mathrm{H}}\right\}$ [6, 7,31-33]. The envelope of $\mathbf{z}$ is modulated by texture enhancement factor $\sigma \in \mathbb{R}$, which is gamma distributed with parameters $v$ and $\mu$, and its probability density function (PDF) is given by [31] as

$$
p_{\sigma}(\sigma)=\frac{1}{\Gamma(v)}\left(\frac{v}{\mu}\right)^{v} \tau^{(v-1)} \exp \left(-\left(\frac{v}{\mu}\right) \sigma\right), \quad \sigma \geq 0 .
$$

For a given value of texture enhancement $\sigma$, and for $M$-dimensional vector $\mathbf{z}, \mathrm{PDF}$ of $\mathbf{c}$ is given as

$$
p_{\mathbf{c} \mid \sigma}(\mathbf{c} \mid \sigma)=\frac{1}{\pi^{M}\left|\Sigma_{\mathbf{c}|\sigma|}\right|} \exp \left(-\mathbf{c}^{\mathrm{H}} \boldsymbol{\Sigma}_{\mathbf{c} \mid \sigma}^{-1} \mathbf{c}\right)
$$

where, $|\cdot|$ represents the matrix determinant operator, and $\boldsymbol{\Sigma}_{\mathbf{c} \mid \sigma}$ is given by

$$
\boldsymbol{\Sigma}_{\mathbf{c} \mid \sigma}=\mathbb{E}\left\{\mathbf{c c}^{\mathrm{H}} \mid \sigma\right\}=\mathbb{E}\left\{\sqrt{\sigma} \mathbf{z} \sqrt{\sigma} \mathbf{z}^{\mathrm{H}}\right\}=\sigma \boldsymbol{\Sigma}_{\mathbf{z z}} .
$$

Substituting (19) in (18) yields the final expression for the PDF of $\mathbf{c}$ given $\sigma$ as,

$$
p_{\mathbf{c} \mid \sigma}(\mathbf{c} \mid \sigma)=\frac{1}{\pi^{M} \sigma^{M}\left|\boldsymbol{\Sigma}_{\mathbf{z z}}\right|} \exp \left(-\frac{\mathbf{c}^{\mathrm{H}} \boldsymbol{\Sigma}_{\mathbf{z z}}^{-1} \mathbf{c}}{\sigma}\right) .
$$

Finally, expression for the PDF of K-distributed sea clutter (c) is obtained by averaging (20) with respect to $\sigma$ and is expressed as

$$
p(\mathbf{c})=\int_{0}^{\infty} p_{\mathbf{c} \mid \sigma}(\mathbf{c} \mid \sigma) p_{\sigma}(\sigma) \mathrm{d} \sigma .
$$

\subsection{Modified Target Detection Test}

In this section, for target detection, we propose a suboptimal GLRT based detector. The detector described in [34], proposed detection of the perfectly known signal by considering the unknown texture enhancement factor $\sigma$, and the known speckle component covariance matrix $\boldsymbol{\Sigma}_{\mathbf{z z}}$. From (14), due to unknown scattering coefficient $\mathbf{X}_{t}$, the signal is not perfectly known at the receiver. Additionally, the covariance matrix $\boldsymbol{\Sigma}_{\mathbf{z z}}$ is also unknown. As the proposed system model (15) has unknown but deterministic $\mathbf{X}_{t}$, and it is applied over both the estimated radar return data and simulated data, the detection test is modified by replacing the unknown $\mathbf{X}_{t}$ in GLRT by its LS estimate followed by the replacement of $\boldsymbol{\Sigma}_{\mathbf{z z}}$ with its maximum likelihood (ML) estimate.

To perform detection test, we consider two hypothesis, $\mathcal{H}_{0}$ for target absent, and $\mathcal{H}_{1}$ for target present. Hence, after applying the assumption of sea clutter dominance over the thermal noise, (15) yields

$$
\begin{aligned}
& \mathcal{H}_{1}: \mathbf{r}_{\mathrm{v}}=\mathbf{p}_{\mathrm{v}}+\mathbf{c}_{\mathrm{v}}, \\
& \mathcal{H}_{0}: \mathbf{r}_{\mathrm{v}}=\mathbf{c}_{\mathrm{v}}
\end{aligned}
$$

where $\mathbf{r}_{\mathrm{v}}=\operatorname{vec}(\mathbf{R}), \mathbf{p}_{\mathrm{v}}=\operatorname{vec}\left(\mathbf{A} \mathbf{X}_{t} \boldsymbol{\Phi}\right)$, and $\mathbf{c}_{\mathrm{v}}=\operatorname{vec}(\mathbf{C})$.
Test statistics $\Lambda(\mathbf{r})$ is the ratio of likelihood of $\mathbf{r}_{\mathrm{v}}$ for two different hypothesis $\mathcal{H}_{0}$ and $\mathcal{H}_{1}$.

$$
\Lambda\left(\mathbf{r}_{\mathrm{v}}\right)=\frac{\mathcal{P}\left(\mathbf{r}_{\mathrm{v}} ; \hat{\mathbf{X}}_{t} ; \hat{\boldsymbol{\Sigma}}_{1}, \mathcal{H}_{1}\right)}{\mathcal{P}\left(\mathbf{r}_{\mathrm{v}} ; \hat{\boldsymbol{\Sigma}}_{0}, \mathcal{H}_{0}\right)} \underset{\mathcal{H}_{0}}{\stackrel{\mathcal{H}_{1}}{\gtrless}} \lambda
$$

where $\mathcal{P}\left(\mathbf{r}_{\mathrm{v}} ; \hat{\mathbf{X}}_{t} ; \hat{\boldsymbol{\Sigma}}_{1}, \mathcal{H}_{1}\right)^{1}$ is the PDF of $\mathbf{r}_{\mathrm{v}}$ under hypothesis $\mathcal{H}_{1}$. Since under hypothesis $\mathcal{H}_{1}, \mathbf{X}_{t}$ and $\boldsymbol{\Sigma}_{1}$ are unknown, $\mathcal{P}\left(\mathbf{r}_{\mathrm{v}} ; \hat{\mathbf{X}}_{t} ; \hat{\boldsymbol{\Sigma}}_{1}, \mathcal{H}_{1}\right)$ is parametrized by the estimates of $\mathbf{X}_{t}$ and $\boldsymbol{\Sigma}_{1}$. Similarly, $\mathcal{P}\left(\mathbf{r}_{\mathrm{v}} ; \hat{\boldsymbol{\Sigma}}_{0}, \mathcal{H}_{0}\right)$ is the PDF of $\mathbf{r}_{\mathrm{v}}$ under hypothesis $\mathcal{H}_{0}$. Similar to case with hypothesis $\mathcal{H}_{1}$, since under hypothesis $\mathcal{H}_{0}, \boldsymbol{\Sigma}_{0}$ is unknown, the $\operatorname{PDF}\left(\mathcal{P}\left(\mathbf{r}_{\mathrm{v}} ; \hat{\boldsymbol{\Sigma}}_{0}, \mathcal{H}_{0}\right)\right)$ is parametrized by the estimates of $\boldsymbol{\Sigma}_{0}$. Further, $\hat{\mathbf{X}}_{t}$ is the LS estimate of scattering coefficient matrix $\mathbf{X}_{t}$. The $\hat{\boldsymbol{\Sigma}}_{1}$, and $\hat{\boldsymbol{\Sigma}}_{0}$ are the ML estimates of covariance matrices $\boldsymbol{\Sigma}_{1}$, and $\boldsymbol{\Sigma}_{0}$, respectively.

As no close form expression for $\mathcal{P}\left(\mathbf{r}_{\mathrm{v}} ; \hat{\mathbf{X}}_{t} ; \hat{\mathbf{\Sigma}}_{1}, \mathcal{H}_{1}\right)$ and $\mathcal{P}\left(\mathbf{r}_{\mathrm{v}} ; \hat{\mathbf{\Sigma}}_{0}, \mathcal{H}_{0}\right)$ is available, (23) can be further simplified using (21) as

$$
\Lambda\left(\mathbf{r}_{\mathrm{v}}\right)=\frac{\int_{0}^{\infty} \mathcal{P}\left(\mathbf{r}_{\mathrm{v}} \mid \sigma ; \hat{\mathbf{X}}_{t} ; \hat{\boldsymbol{\Sigma}}_{1 \mid \sigma}, \mathcal{H}_{1}\right) \mathcal{P}_{\sigma}(\sigma) \mathrm{d} \sigma}{\int_{0}^{\infty} \mathcal{P}\left(\mathbf{r}_{\mathrm{v}} \mid \sigma ; \hat{\boldsymbol{\Sigma}}_{0 \mid \sigma}, \mathcal{H}_{0}\right) \mathcal{P}_{\sigma}(\sigma) \mathrm{d} \sigma} \underset{\mathcal{H}_{0}}{\stackrel{\mathcal{H}_{1}}{\gtrless}} \lambda
$$

where $\mathcal{P}\left(\mathbf{r}_{\mathrm{v}} \mid \sigma ; \hat{\mathbf{X}}_{t} ; \hat{\boldsymbol{\Sigma}}_{1 \mid \sigma}, \mathcal{H}_{1}\right)$ and $\mathcal{P}\left(\mathbf{r}_{\mathrm{v}} \mid \sigma ; \hat{\boldsymbol{\Sigma}}_{0 \mid \sigma}, \mathcal{H}_{0}\right)$ are the conditional PDF of $\mathbf{r}_{\mathrm{v}}$ under hypothesis $\mathcal{H}_{1}$ and $\mathcal{H}_{0}$, respectively, conditioned on $\sigma$.

From [34], for a known covariance matrix $\boldsymbol{\Sigma}_{\mathbf{z z}}$, the $\hat{\mathbf{\Sigma}}_{0 \mid \sigma}=\hat{\sigma}_{0} \boldsymbol{\Sigma}_{\mathrm{zz}}$, and $\hat{\boldsymbol{\Sigma}}_{1 \mid \sigma}=\hat{\sigma}_{1} \boldsymbol{\Sigma}_{\mathrm{zz}}$. Since, $\mathbf{z}$ is Gaussian distributed correlated random process $[31,34,35]$, the $\hat{\sigma}_{0}$ and $\hat{\sigma}_{1}$ are considered to be the ML estimates of the unknown clutter powers. The same are estimated by considering the following two likelihood functions

$$
\begin{gathered}
\mathcal{P}\left(\mathbf{r}_{\mathrm{v}} \mid \sigma_{0} ; \mathcal{H}_{0}\right)=\frac{1}{\pi^{M} \sigma_{0}^{M}\left|\boldsymbol{\Sigma}_{\mathbf{z z}}\right|} \exp \left(\frac{-\mathbf{r}_{\mathrm{v}}^{\mathrm{H}} \mathbf{\Sigma}_{\mathbf{z z}}^{-1} \mathbf{r}_{\mathrm{v}}}{\sigma_{0}}\right), \\
\mathcal{P}\left(\mathbf{r}_{\mathrm{v}} \mid \sigma_{1} ; \mathcal{H}_{1}\right)= \\
\frac{1}{\pi^{M} \sigma_{1}^{M}\left|\boldsymbol{\Sigma}_{\mathbf{z z}}\right|} \exp \left(\frac{-\left(\mathbf{r}_{\mathrm{v}}-\mathbf{p}_{\mathrm{v}}\right)^{\mathrm{H}} \boldsymbol{\Sigma}_{\mathbf{z z}}^{-1}\left(\mathbf{r}_{\mathrm{v}}-\mathbf{p}_{\mathrm{v}}\right)}{\sigma_{1}}\right) .
\end{gathered}
$$

Hence, ML estimate of $\sigma_{0}$ and $\sigma_{1}$ is given by

$$
\begin{gathered}
\hat{\sigma}_{0}=\frac{1}{M} \mathbf{r}_{\mathrm{v}}^{\mathrm{H}} \mathbf{\Sigma}_{\mathbf{z z}}^{-1} \mathbf{r}_{\mathrm{v}}, \\
\hat{\sigma}_{1}=\frac{1}{M}\left(\mathbf{r}_{\mathrm{v}}-\mathbf{p}_{\mathrm{v}}\right)^{\mathrm{H}} \boldsymbol{\Sigma}_{\mathbf{z z}}^{-1}\left(\mathbf{r}_{\mathrm{v}}-\mathbf{p}_{\mathrm{v}}\right) .
\end{gathered}
$$

Using (24), (25), and (26), the test statistics $\Lambda\left(\mathbf{r}_{\mathrm{v}}\right)$ is given by

$$
\begin{aligned}
\Lambda\left(\mathbf{r}_{\mathrm{v}}\right) \in \mathbb{R} & =\left(\frac{\hat{\sigma}_{0}}{\hat{\sigma}_{1}}\right)^{M} \underset{\mathcal{H}_{0}}{\stackrel{\mathcal{H}_{1}}{\gtrless}} \lambda, \\
& =\left(\frac{\hat{\sigma}_{0}}{\hat{\sigma}_{1}}\right) \underset{\mathcal{H}_{0}}{\stackrel{\mathcal{H}_{1}}{\gtrless}} \lambda^{\prime}
\end{aligned}
$$

where $\lambda^{\prime}=(\lambda)^{\frac{1}{M}}$.

${ }^{1}$ the semicolon (;) is used to represent parametrization, and comma "," represents under a hypothesis. 
Substituting (26) in (27), yields the final expression for test statistics as

$$
\Lambda\left(\mathbf{r}_{\mathrm{v}}\right)=\frac{\mathbf{r}_{\mathrm{v}}^{\mathrm{H}} \hat{\mathbf{\Sigma}}_{\mathbf{z z}}^{-1} \mathbf{r}_{\mathrm{v}}}{\left(\mathbf{r}_{\mathrm{v}}-\operatorname{vec}\left(\mathbf{A} \hat{\mathbf{X}}_{t} \boldsymbol{\Phi}\right)\right)^{\mathrm{H}} \hat{\boldsymbol{\Sigma}}_{\mathbf{z z}}^{-1}\left(\mathbf{r}_{\mathrm{v}}-\operatorname{vec}\left(\mathbf{A} \hat{\mathbf{X}}_{t} \boldsymbol{\Phi}\right)\right)} \underset{\mathcal{H}_{0}}{\stackrel{\mathcal{H}_{1}}{\gtrless}} \lambda^{\prime}
$$

where $\hat{\boldsymbol{\Sigma}}_{\mathbf{z z}}=\frac{1}{I} \sum_{i=1}^{I}\left(\frac{M}{\mathbf{r}_{\mathrm{v}}^{i}{ }_{\mathbf{r}_{\mathrm{v}}^{i}}^{i}}\right) \mathbf{r}_{\mathrm{v}}^{i} \mathbf{r}_{\mathrm{v}}^{i \mathrm{H}}$ is the ML estimate of speckle covariance matrix $\boldsymbol{\Sigma}_{\mathbf{z z}}$ obtained from the $I$ observations of $\mathbf{r}_{\mathrm{v}}$ from different range gates under hypothesis $\mathcal{H}_{0}$, and $\hat{\mathbf{X}}_{t}=\operatorname{diag}\left(\operatorname{diag}\left(\mathbf{A}^{-1} \mathbf{R} \boldsymbol{\Phi}^{\mathrm{H}}\left(\boldsymbol{\Phi} \boldsymbol{\Phi}^{\mathrm{H}}\right)^{-1}\right)\right)$ is the LS estimate of $\mathbf{X}_{t}$.

\subsection{Analysis of Proposed Detector}

From (28), we can analyse that for a given clutter power $\mu \operatorname{tr}\left(\boldsymbol{\Sigma}_{\mathbf{z z}}\right)$, better estimate of $\mathbf{X}_{t}$ yields higher value of test statistics $\Lambda\left(\mathbf{r}_{\mathrm{v}}\right)$ which in turn improves the proposed detector's performance. In (28), for fixed signal-to-clutter ratio (SCR), increasing $L$ yields better estimate of $\mathbf{X}_{t}\left(\hat{\mathbf{X}}_{t}\right)$. Consequently, as $\hat{\mathbf{X}}_{t}$ approaches $\mathbf{X}_{t}$, the denominator in (28) reduces further, thereby increasing the value of $\Lambda\left(\mathbf{r}_{\mathrm{v}}\right)$. Subsequently, $\Lambda\left(\mathbf{r}_{\mathrm{v}}\right)$ crosses $\lambda^{\prime}$ more number of times, and hence results in better target detection. Therefore, for same $P_{\mathrm{FA}}$ and $\lambda$, in addition to providing frequency diversity, the OFDM waveform provides additional information about the target from multiple scattering centers, which resonate differently at different sub-carrier frequency [10].

Further, a closed-form expression for $P_{\mathrm{D}}$ and $P_{\mathrm{FA}}$ for Kdistributed clutter is difficult to achieve, hence the sea clutter is assumed to be uncorrelated Gaussian distributed. This assumption is feasible for a very high value of shape parameter $v$, as given in [31] and shown in (17) generally for $v \gtrsim 20$, the PDF $p_{\sigma}(\sigma)$ can be denoted as a Dirac function concentrated around the deterministic value $\sigma=\mu$. Consequently, as $p_{\mathbf{c}_{\mathrm{v}}}\left(\mathbf{c}_{\mathrm{v}}\right)=\int_{0}^{\infty} p_{\mathbf{c}_{\mathrm{v}} \mid \sigma}\left(\mathbf{c}_{\mathrm{v}} \mid \sigma\right) p_{\sigma}(\sigma) \mathrm{d} \sigma$, the clutter PDF $p_{\mathbf{c}_{\mathrm{v}}}\left(\mathbf{c}_{\mathrm{v}}\right)$ reduces to multivariate Gaussian. For mathematical tractability and simplicity of theoretical analysis, the test statistics in (27) is represented as

$$
\begin{aligned}
\Lambda^{\prime}\left(\mathbf{r}_{\mathrm{v}}\right) & =\Lambda\left(\mathbf{r}_{\mathrm{v}}\right)^{\frac{1}{M}}-1, \\
& =\left(\frac{\hat{\sigma}_{0}}{\hat{\sigma}_{1}}\right)-1 .
\end{aligned}
$$

For Gaussian distributed sea clutter, the ML estimates $\left(\hat{\sigma}_{0}\right.$ and $\left.\hat{\sigma}_{1}\right)$ are

$$
\begin{aligned}
& \hat{\sigma}_{0}=\frac{1}{M} \operatorname{tr}\left(\mathbf{R}^{\mathrm{H}} \mathbf{R}\right) . \\
& \hat{\sigma}_{1}=\frac{1}{M} \operatorname{tr}\left(\left(\mathbf{R}-\mathbf{A} \mathbf{X}_{t} \boldsymbol{\Phi}\right)^{\mathrm{H}}\left(\mathbf{R}-\mathbf{A} \mathbf{X}_{t} \boldsymbol{\Phi}\right)\right) .
\end{aligned}
$$

From (29), (30) and (31) we get

$$
\Lambda^{\prime}(\mathbf{R})=\frac{\operatorname{tr}\left(\mathbf{R}^{\mathrm{H}} \mathbf{P R}\right)}{\operatorname{tr}\left(\mathbf{R}^{\mathrm{H}} \mathbf{P}_{\perp} \mathbf{R}\right)}
$$

where $\mathbf{P}=\boldsymbol{\Phi}^{\mathrm{H}}\left(\boldsymbol{\Phi} \boldsymbol{\Phi}^{\mathrm{H}}\right)^{-1} \boldsymbol{\Phi}$ is the projection matrix, and $\mathbf{P}_{\perp}$ is the orthogonal projection matrix related to $\mathbf{P}$ as $\mathbf{P}_{\perp}=\mathbf{I}-\mathbf{P}$ (I is an identity matrix of dimension $M \times M$ ).

The analytical expressions for $P_{\mathrm{D}}$ and $P_{\mathrm{FA}}$ for the test statistics (32) are given by the following relations

$$
\begin{gathered}
P_{\mathrm{D}}=\int_{\lambda_{\mathrm{g}}}^{\infty} \mathcal{P}_{\mathcal{H}_{1}}\left(\Lambda^{\prime}(\mathbf{R})\right) \mathrm{d} \Lambda^{\prime}(\mathbf{R}), \\
P_{\mathrm{FA}}=\int_{\lambda_{\mathrm{g}}}^{\infty} \mathcal{P}_{\mathcal{H}_{0}}\left(\Lambda^{\prime}(\mathbf{R})\right) \mathrm{d} \Lambda^{\prime}(\mathbf{R})
\end{gathered}
$$

where $\lambda_{\mathrm{g}}$ is the detection threshold, $\mathcal{P}_{\mathcal{H}_{1}}\left(\Lambda^{\prime}(\mathbf{R})\right)$ is the PDF of $\Lambda^{\prime}(\mathbf{R})$ under hypothesis $\mathcal{H}_{1}$, and $\mathcal{P}_{\mathcal{H}_{0}}\left(\Lambda^{\prime}(\mathbf{R})\right)$ is the PDF of $\Lambda^{\prime}(\mathbf{R})$ under hypothesis $\mathcal{H}_{0}$.

Under Gaussian assumption for sea clutter, both the numerator and the denominator of (32) under hypothesis $\mathcal{H}_{0}$ are central Chi-Squared distributed random variables

$$
\begin{array}{rlll}
\operatorname{tr}\left(\mathbf{R}^{\mathrm{H}} \mathbf{P R}\right) \sim \chi_{v_{1}}^{2} & \text { under } & \mathcal{H}_{0}, \\
\operatorname{tr}\left(\mathbf{R}^{\mathrm{H}} \mathbf{P}_{\perp} \mathbf{R}\right) \sim \chi_{v_{2}}^{2} & \text { under } & \mathcal{H}_{0}
\end{array}
$$

where $v_{1}=2 L \mathcal{R}(\mathcal{P})$ and $v_{2}=2 L \mathcal{R}\left(\mathcal{P}_{\perp}\right)$ are the degrees of freedom for numerator and denominator respectively. Since, the ratio of central Chi-Squared distributed random variable follows central $F_{v_{1}, v_{2}}$ distribution, hence under $\mathcal{H}_{0}, \Lambda^{\prime}(\mathbf{R})$ is distributed as

$$
\Lambda^{\prime}(\mathbf{R}) \sim F_{v_{1}, v_{2}} .
$$

Using (34), the expression for $P_{\mathrm{FA}}$ can be defined in terms of the right tail probability $\left(Q_{F_{v_{1}, v_{2}}}\left(\lambda_{\mathrm{g}}\right)\right)$ of $F_{v_{1}, v_{2}}$ as

$$
P_{\mathrm{FA}}=Q_{F_{v_{1}, v 2}}\left(\lambda_{\mathrm{g}}\right)
$$

where

$$
Q_{F_{v_{1}, v_{2}}}\left(\lambda_{\mathrm{g}}\right)=\int_{\lambda_{\mathrm{g}}}^{\infty} \frac{\left(\frac{v_{1}}{v_{2}}\right)^{\frac{v_{1}}{2}} \Lambda^{\prime}(\mathbf{R})^{\frac{v_{1}}{2}-1}}{B\left(\frac{v_{1}}{2}, \frac{v_{2}}{2}\right)\left(1+\frac{v_{1}}{v_{2}} \Lambda^{\prime}(\mathbf{R})\right)^{\frac{v_{1}+v_{2}}{2}}} \mathrm{~d} \Lambda^{\prime}(\mathbf{R})
$$

and $B$ represents Beta function. Hence, the theoretical value of $P_{\mathrm{FA}}$ is obtained by solving (39) numerically with the method proposed in [36].

Contrary to the case under hypothesis $\mathcal{H}_{0}$, numerator of (32) under hypothesis $\mathcal{H}_{1}$ is non-central Chi-Squared distributed random variable $\left(\chi_{v_{1}}^{\prime 2}(\delta)\right)$ with a non-centrality parameter $\delta=\operatorname{tr}\left\{\left(\mathbf{A} \mathbf{X}_{t} \boldsymbol{\Phi}\right)\left(\mathbf{A} \mathbf{X}_{t} \boldsymbol{\Phi}\right)^{\mathrm{H}}\right\}$, and the denominator is central Chi-Squared distributed random variable $\left(\chi_{v_{2}}^{2}\right)$. Hence, their distribution are as follows:

$$
\begin{aligned}
& \operatorname{tr}\left(\mathbf{R}^{\mathrm{H}} \mathbf{P R}\right) \sim \chi_{v_{1}}^{\prime 2}(\delta) \quad \text { under } \quad \mathcal{H}_{1}, \\
& \operatorname{tr}\left(\mathbf{R}^{\mathrm{H}} \mathbf{P}_{\perp} \mathbf{R}\right) \sim \chi_{v_{2}}^{2} \quad \text { under } \mathcal{H}_{1} \text {. }
\end{aligned}
$$


Since the ratio of non-central and central Chi-squared distributed random variables is non-central $F^{\prime}{ }_{v_{1}, v_{2}}(\delta)$ distributed, under hypothesis $\mathcal{H}_{1}, \Lambda^{\prime}(\mathbf{R})$ is distributed as

$$
\Lambda^{\prime}(\mathbf{R}) \sim F^{\prime}{ }_{v_{1}, v_{2}}(\delta) .
$$

Thus $P_{\mathrm{D}}$ as shown in (33) can be written as the right tail probability $\left(Q_{F^{\prime}{ }_{v_{1}, v_{2}}(\delta)}\left(\lambda_{\mathrm{g}}\right)\right)$ of ${F^{\prime}}_{v_{1}, v_{2}}(\delta)$

$$
P_{\mathrm{D}}=Q_{F^{\prime}{ }_{v_{1}, v_{2}}(\delta)}\left(\lambda_{\mathrm{g}}\right)
$$

$$
\begin{aligned}
& \text { where } \\
& \begin{aligned}
Q_{F^{\prime}{ }_{{ }_{1}, v_{2}}(\delta)}\left(\lambda_{\mathrm{g}}\right)= & \int_{\lambda_{\mathrm{g}}}^{\infty} \exp \left(\frac{-\delta}{2}\right) \sum_{k=0}^{k=\infty} \frac{(\delta / 2)^{k}}{k !} \frac{\left(\frac{v_{1}}{v_{2}}\right)^{\frac{1}{2} v_{1}+k}}{B\left(\frac{v_{1}+2 k}{2}, \frac{v_{2}}{2}\right)} \\
& \Lambda^{\prime}(\mathbf{R})^{\frac{v_{1}}{2}+k-1}\left(1+\frac{v_{1}}{v_{2}} \Lambda^{\prime}(\mathbf{R})\right)^{\frac{-1}{2}\left(v_{1}+v_{2}\right)-k} \\
& \mathrm{~d} \Lambda^{\prime}(\mathbf{R}) .
\end{aligned}
\end{aligned}
$$

Similar to $P_{\mathrm{FA}}$ as shown in (39), theoretical values of $P_{\mathrm{D}}$ for different values of detection threshold $\lambda_{\mathrm{g}}$ and $L$ is obtained by solving (44) numerically.

Hence, from (39) and (44), the analytical expression for ROC of (32), parametrized by $\lambda_{\mathrm{g}}$ and $L$ is given by

$$
P_{\mathrm{D}}=Q_{F^{\prime}{ }_{v_{1}, v_{2}}(\delta)}\left(Q_{F_{v_{1}, v 2}}^{-1}\left(P_{\mathrm{FA}}\right)\right) .
$$

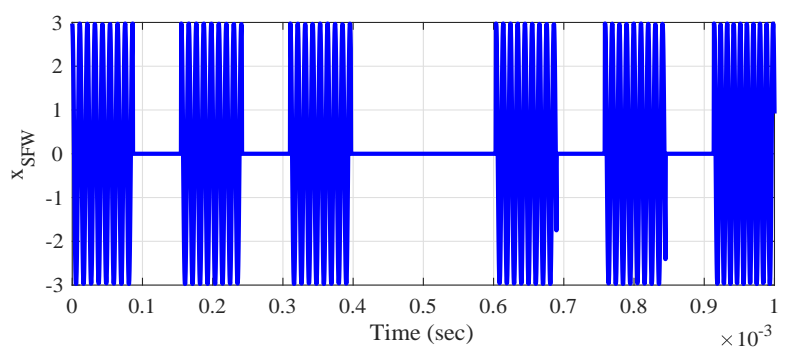

Fig. 2. Burst of 25 pulses for one CPI representing the group of stepped frequency pulses $\left(x_{\mathrm{SFW}}\right)$.

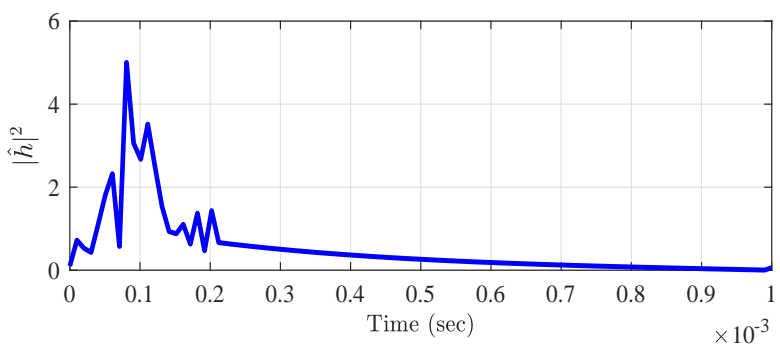

Fig. 4. Amplitude square of estimated radar system IR coefficients $|\hat{h}|^{2}$.

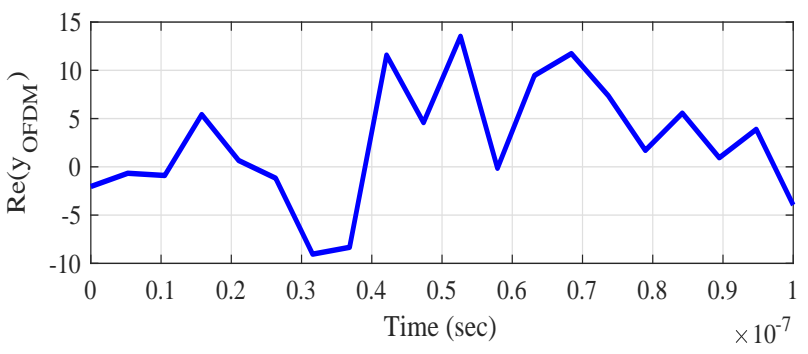

Fig. 6. Real part of an estimated radar return ( $\left.y_{\text {OFDM }}\right)$ for $L=4$.

\section{Simulation Results}

In this section, simulation results to validate the proposed method of estimating OFDM radar return data and performance analysis of the proposed detector for estimated and simulated data are described in detail.

\subsection{Estimation of OFDM Radar Return Data}

Simulations for estimation of OFDM radar return is performed in two steps. In the first step, IR $\mathbf{h}$ of the radar system is estimated by LS using the generated input signal $x_{\mathrm{SFW}}(n)$ and the output signal $y_{\mathrm{SFw}}(n)$ depicted in Fig. 2 and Fig. 3, respectively. For simulations, $y_{\mathrm{SFw}}(n)$ is taken from a single CPI of the original radar return data set from the CSIR 2006 OTB 2006 Measurement Trial [1], and $x_{\text {SFw }}(n)$ is generated according to the specifications of the transmitted SF waveform, provided with the data sets and described in Tab. 1. Estimated radar system IR for a single CPI is shown in Fig. 4. The OFDM radar return data $y_{\text {OFDM }}(n)$ is estimated by observing response of the radar system for generated OFDM waveform $x_{\mathrm{OFDM}}(n)$ as shown in Fig. 5 for four sub-carriers. Specifications for $x_{\text {OFDM }}(n)$ is given in Tab. 2. Particularly, for OFDM waveform for $L=4$ and maximum $L=32$, estimated OFDM radar return data $\left(y_{\text {OFDM }}(n)\right)$ is shown in Fig. 6 and Fig. 7, respectively.

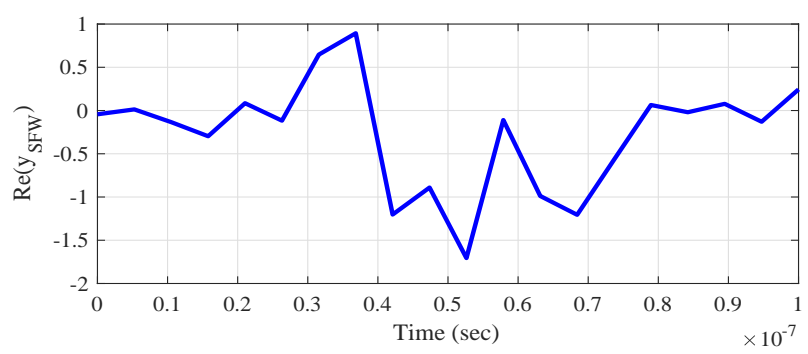

Fig. 3. Real part of recorded radar return $\left(y_{\mathrm{SFW}}\right)$ for SF waveform for single PRI.

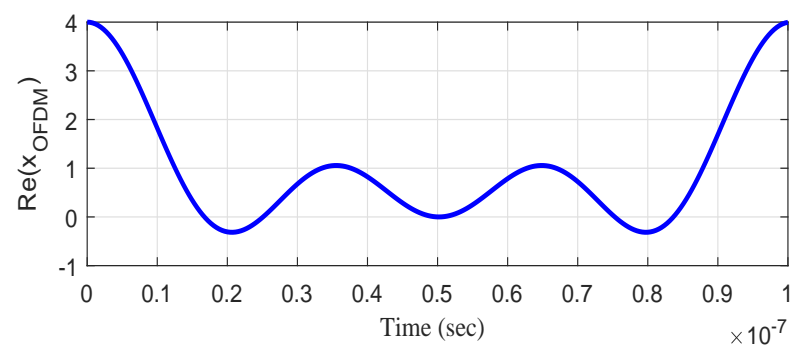

Fig. 5. Real part of Incorporated OFDM waveform $\left(x_{\text {OFDM }}\right)$ for one single PRI.

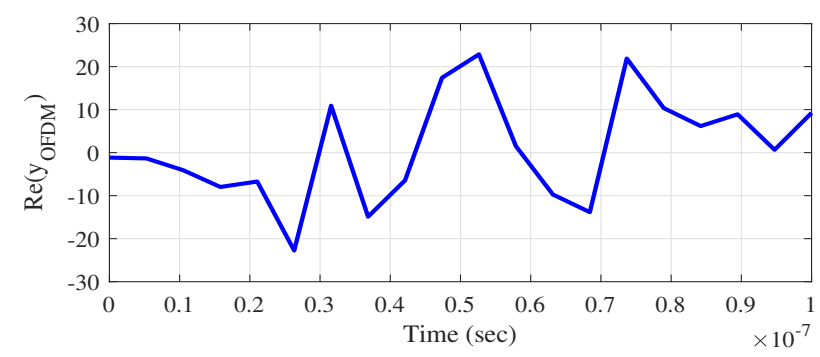

Fig. 7. Real part of an estimated radar return ( $\left.y_{\text {OFDM }}\right)$ for $L=32$. 


\begin{tabular}{|c|c|}
\hline Transmitted waveform type & SF waveform \\
\hline Number of frequency channels & 25 \\
\hline Frequency interval & $8.9 \mathrm{GHz}$ to $9.02 \mathrm{GHz}$ \\
\hline Frequency step size & $5 \mathrm{MHz}$ \\
\hline Center frequency $\left(f_{\mathrm{c}}\right)$ & $9 \mathrm{GHz}$ \\
\hline Bandwidth $(B)$ & $5 \mathrm{MHz}$ \\
\hline Pulse duration $\left(T_{\mathrm{SF}}\right)$ & $0.1 \mu \mathrm{s}$ \\
\hline Pulse repetition interval $\left(T_{\mathrm{PRI}}\right)$ & $0.04 \mathrm{~ms}$ \\
\hline Sampling frequency $\left(f_{\mathrm{S}}=725 \mathrm{~B}\right)$ & $3.625 \mathrm{GHz}$ \\
\hline
\end{tabular}

Tab. 1. Specifications for SF waveform $\left(x_{\mathrm{SFW}}\right)$.

\begin{tabular}{|c|c|}
\hline Transmitted waveform type & OFDM \\
\hline Number of sub-carriers $(L)$ & $2^{l} ; l=2,3,4,5$ \\
\hline Bandwidth $(B)$ & $125 \mathrm{MHz}$ \\
\hline Subcarrier spacing $\left(\Delta f=\frac{B}{L}\right)$ & $31.25 \mathrm{MHz}-3.9063 \mathrm{MHz}$ \\
\hline Center frequency $\left(f_{\mathrm{c}}\right)$ & $9 \mathrm{GHz}$ \\
\hline Pulse duration $\left(T_{\mathrm{O}}=\frac{1}{\Delta f}\right)$ & $0.032 \mu \mathrm{s}-0.256 \mu \mathrm{s}$ \\
\hline Pulse repetition interval $\left(T_{\mathrm{PRI}}\right)$ & $0.04 \mathrm{~ms}$ \\
\hline Sampling frequency $\left(f_{\mathrm{S}}=25 B\right)$ & $3.125 \mathrm{GHz}$ \\
\hline
\end{tabular}

Tab. 2. Specifications for OFDM waveform $\left(x_{\mathrm{OFDM}}\right)$.

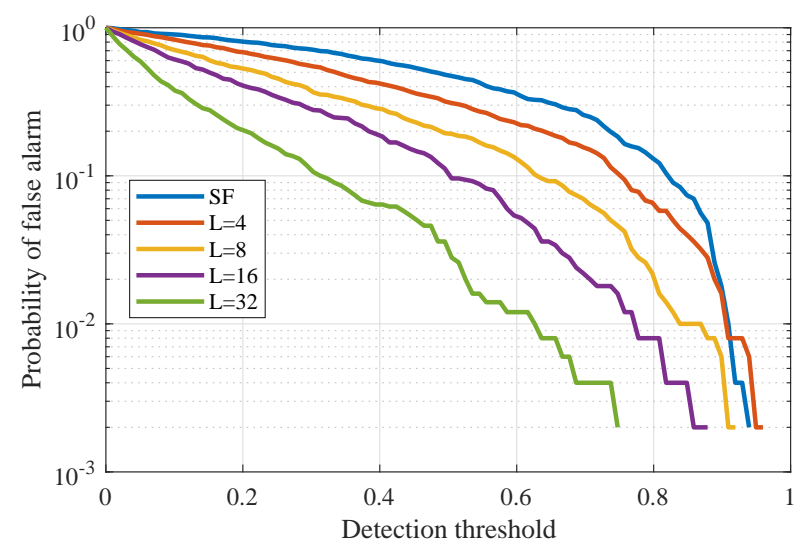

Fig. 8. Probability of false alarm for SF waveform and OFDM waveform comprises different number of sub-carriers.

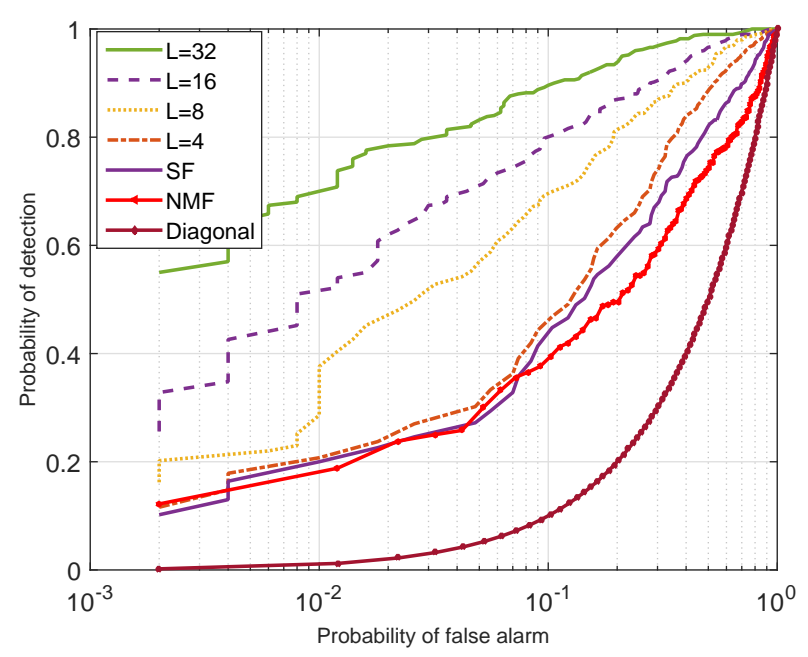

Fig. 9. ROC of proposed detector test statistics and conventional NMF detector.

\subsection{Detector Performance for Estimated OFDM Radar Return}

Performance of the proposed methodology of incorporating OFDM waveform in radar systems is examined by analyzing the performance of the detection test given by (28). The expected range of required detection threshold $\lambda$ for which the detection test is evaluated is calculated by utilizing the data from the observation corresponding to hypothesis $\mathcal{H}_{0}$. The comparative plot for $P_{\mathrm{FA}}$ for different values of $\lambda$ corresponding to SF waveform and different OFDM subscribers is shown in Fig. 8. The decrease in $P_{\mathrm{FA}}$ as $L$ increases is observed in Fig. 8, this reflects an improvement in the performance of target detection test by exploiting the frequency diversity of an OFDM waveform. Moreover, as shown in Fig. 9, in the case of OFDM radar, receiving echoes for each OFDM sub-carrier separately provide an additional "look" at the target, resulting in improved target detection capability over SF radar and conventional NMF. Furthermore, from Fig. 9, at $P_{\mathrm{FA}}=10^{-2}$, the $P_{\mathrm{D}}$ achieved by SF radar and NMF is 0.2 , and for the OFDM radar $P_{\mathrm{D}}$ ranges from 0.2 to 0.75 , hence better $P_{\mathrm{D}}$ is obtained in the case of OFDM radar, which can further be enhanced by varying the number of subcarriers. Effect of the high resolution and frequency diversity attained by an OFDM waveform is reflected by a decrease in $P_{\mathrm{FA}}$ and improvement in $P_{\mathrm{D}}$, as shown in Figs. 8-9 (Please note that, since the real data, which is limited in number, is used the resulting plots are not smooth).

\subsection{Detector Performance for Simulated OFDM Radar Return}

The performance improvement observed by utilizing estimated OFDM radar return is validated by running the detection test over simulated data as well as by using (15). For K-distributed clutter, there is no closed form expression that relates $P_{\mathrm{FA}}, \lambda$, and $P_{\mathrm{D}}$, hence, the detection test is done an ensemble for $10^{5}$ Monte Carlo simulations. In (15), $\boldsymbol{\Phi}$ is generated for fixed target Doppler frequency set $f_{\mathrm{D}_{\mathrm{t}}}=\left[f_{0 \mathrm{D}_{\mathrm{t}}}, f_{1 \mathrm{D}_{\mathrm{t}}}, \ldots, f_{(L-1) \mathrm{D}_{\mathrm{t}}}\right]$. The elements of $f_{\mathrm{D}_{\mathrm{t}}}$ takes value from the known Doppler frequency range (Doppler spread) i.e. from $\left\{-\frac{1}{2 T_{\mathrm{PRI}}}, \cdots, \frac{1}{2 T_{\mathrm{PRI}}}\right\}$. The diagonal elements of the matrix A are chosen from the optimized set of phase codes with low PAPR. The values for diagonal elements of $\mathbf{X}_{t}$ is realized from the normal distribution having zero mean and unit variance i.e. $\operatorname{diag}\left(\mathbf{X}_{t}\right) \in \mathcal{N}(\mathbf{0}, \mathbf{I})$. To replicate the sea clutter by which the estimated OFDM radar return data is affected, the K-distributed clutter part of (15) is simulated by utilizing the relation shown in (16). The elements of $\mathbf{z}$ are realized as $\mathbf{z} \in \mathcal{N}\left(\mathbf{0}, \boldsymbol{\Sigma}_{\mathbf{z z}}\right)$, where $\boldsymbol{\Sigma}_{\mathbf{z z}}$ has elements $\left(\Sigma_{\mathbf{z z}}\right)_{i j}=\rho^{|i-j|} \forall i, j \in\{1, \ldots, M\}$, where $\rho$ is the one-lag correlation coefficient. The simulation for obtaining detector performance is run for $M=11$, the SCR defined as $\left(\frac{\mathbf{p}_{\mathrm{v}}^{\mathrm{H}} \mathbf{p}_{\mathrm{v}}}{2 \mu \operatorname{tr}\left(\mathbf{\Sigma}_{\mathrm{zz}}\right)}\right)$ is set at $-10 \mathrm{~dB}$, and $\rho=0.9$. The obtained simulation results for $P_{\mathrm{FA}}$ and ROC are shown in Fig. 10 and Fig. 11, respectively. As observed from Figs. 10-11, detector ROC and $P_{\text {FA }}$ obtained by utilizing the simulated data follow similar trend 
as the detector ROC and $P_{\mathrm{FA}}$ have followed for estimated OFDM radar return data, shown in Figs. 8-9. Particularly, as shown in Fig. 11, proposed detector test statistics surpasses the performance of conventional NMF detector. In Fig. 11, the diagonal line corresponding to $P_{\mathrm{FA}}=P_{\mathrm{D}}$ is shown with line in brown. This analysis validates proposed method for estimating OFDM radar return from the real radar return, since, both the simulated and measured radar return follows a similar trend.

\subsection{Detector Performance under Gaussian Approximation for Sea Clutter}

We demonstrate performance of the detector under the assumption of uncorrelated Gaussian distributed sea clutter.

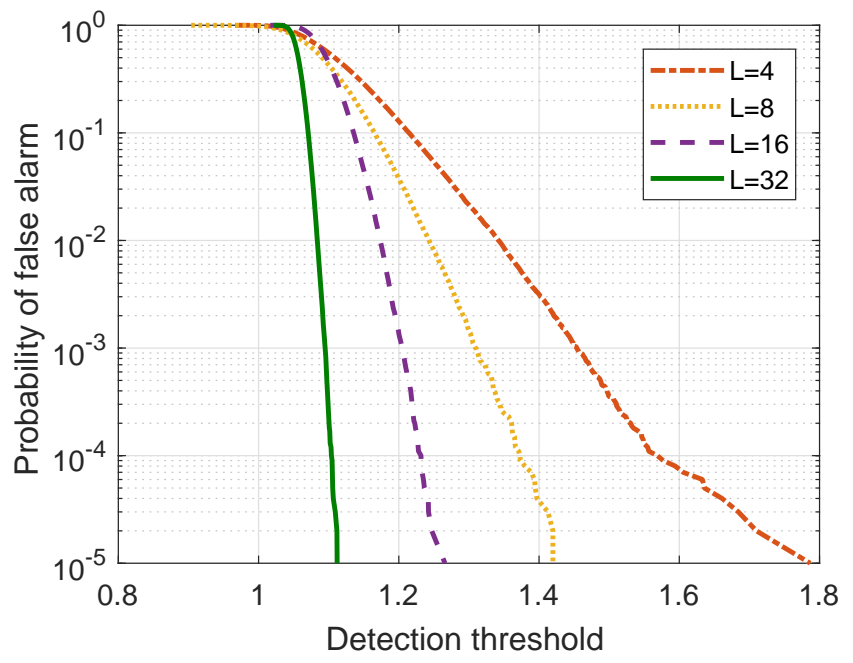

Fig. 10. Probability of false alarm utilizing simulated OFDM radar return data.

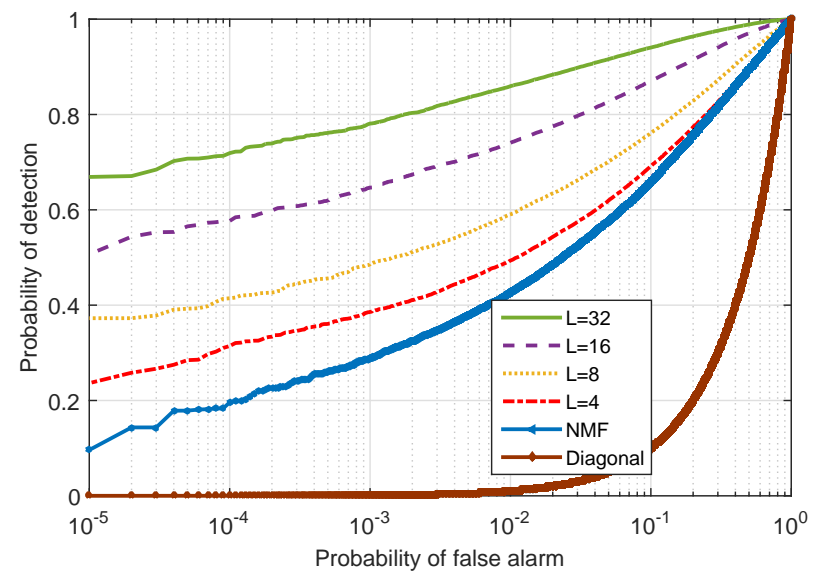

Fig. 11. ROC of proposed detector test statistics utilizing simulated OFDM radar return data and NMF detector.
For this, the detection test derived for a very high value of shape parameter $v$, described by (32) is used. For simulations, signal part of the observations $\mathbf{R}$ is generated as described in Sec. 3 for K-distributed clutter. However, the sea clutter, $\mathbf{C}$, is realized as $\mathbf{C} \in \mathcal{N}\left(\mathbf{0}, \alpha^{2} \mathbf{I}\right)$, where $\alpha^{2}$ is variance of the sea clutter. The SCR is defined as $\frac{\operatorname{tr}\left\{\left(\mathbf{A X} \mathbf{X}_{t} \boldsymbol{\Phi}\right)\left(\mathbf{A} \mathbf{X}_{t} \boldsymbol{\Phi}\right)^{\mathrm{H}}\right\}}{M \alpha^{2}}$ is set at $-10 \mathrm{~dB}$ and $M=11$. As observed from Fig. 12 and Fig. 13, with increase in $L$, both the $P_{\mathrm{FA}}$ and ROC has similar performance improvement as followed by the ROC and $P_{\mathrm{FA}}$ for estimated and simulated OFDM radar return data. Moreover, in Fig. 13, the diagonal line corresponding to $P_{\mathrm{FA}}=P_{\mathrm{D}}$ is shown with line in brown. This analysis of detector performance under Gaussian distributed sea clutter validates the correctness and suitability of the proposed system model and detection test for surveillance in the marine sea environment.

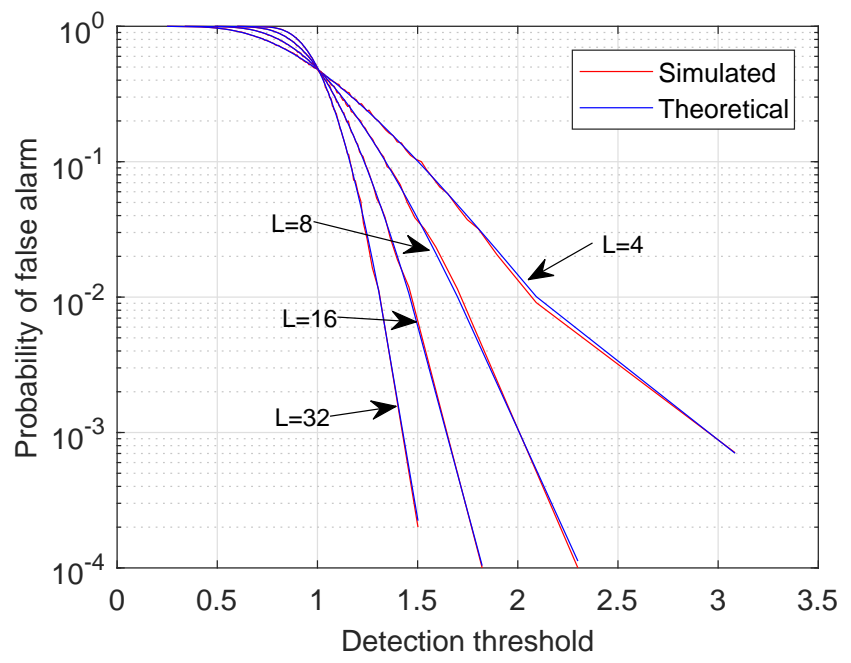

Fig. 12. Probability of false alarm utilizing simulated OFDM radar return data and derived analytical expression under the assumption of Gaussian distributed sea clutter.

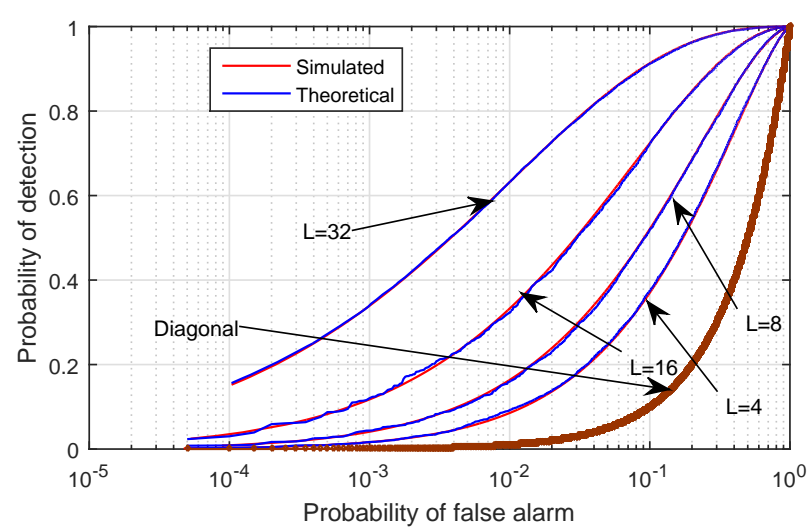

Fig. 13. ROC utilizing simulated OFDM radar return data and derived analytical expression under the assumption of Gaussian distributed sea clutter. 


\section{Conclusion and Future Work}

In this paper, we performed small boat detection in the sea environment using OFDM waveform as a transmitted surveillance waveform. We proposed a method to estimate the OFDM radar return data using CSIR recorded radar return data for SF waveform. System model corresponding to the mathematical representation of radar echoes for OFDM waveform was proposed. The derived system model demonstrates the frequency diversity obtained after employing the OFDM waveform in a radar system. Further, detection of the target utilizing estimated data was done by modified GLRT. Simulation results for $P_{\mathrm{FA}}$ and $P_{\mathrm{D}}$, clearly show improvement in target detection over conventional NMF. The performance is further improved as the number of OFDM sub-carriers increases. The obtained improvement in detector performance with a number of OFDM sub-carriers was validated through analytical expressions of $P_{\mathrm{FA}}$ and $P_{\mathrm{D}}$, and by detector's performance obtained by utilizing simulated data. The demonstrated improvement in detection performance implies the superiority and suitability of OFDM waveform over the conventional radar waveforms.

In the future, the non-linear estimator may be explored for the estimation of OFDM radar return.

\section{Acknowledgments}

This work is an outcome of the research and development work undertaken in the project under the Visvesvaraya PhD Scheme of Ministry of Electronics and Information Technology, Government of India, being implemented by Digital India Corporation.

\section{References}

[1] HERSELMAN, P. L., BAKER, C. J., DE WIND, H. J. An analysis of $\mathrm{X}$-band calibrated sea clutter and small boat reflectivity at mediumto-low grazing angles. International Journal of Navigation and $\mathrm{Ob}$ servation, 2008, vol. 2008, p. 1-14. DOI: 10.1155/2008/347518

[2] HERSELMAN, P. L., DE WIND, H. J. Improved covariance matrix estimation in spectrally inhomogeneous sea clutter with application to adaptive small boat detection. In Proceedings of the IET International Conference on Radar Systems. Adelaide (Australia), 2008, p. 94-99. DOI: 10.1109/RADAR.2008.4653898

[3] HERSELMAN, P. L., BAKER, C. J. Analysis of calibrated sea clutter and boat reflectivity data at C-and X-band in South African coastal waters. In Proceedings of the IET International Conference on Radar Systems. Edinburgh (UK), 2007, p. 1-5. DOI: 10.1049/cp:20070616

[4] WATTS, S. Radar detection prediction in K-distributed sea clutter and thermal noise. IEEE Transactions on Aerospace and Electronic Systems, 1987, vol. 23, no. 1, p. 40-45. DOI: 10.1109/TAES.1987.313334

[5] GINI, F., GRECO, M.V., SANGSTON, K.J., FARINA, A. Coherent adaptive radar detection in non-Gaussian clutter. In Proceedings of the Conference on Signals, Systems and Computers. Pacific Grove (USA), 1997, p. 255-259. DOI: 10.1109/ACSSC.1997.680181
[6] PANAGOPOUlOS, S., SORAGHAN, J. J. Small-target detection in sea clutter. IEEE Transactions on Geoscience and Remote Sensing, 2004, vol. 42, no. 7, p. 1355-1361. DOI: $10.1109 /$ TGRS.2004.827259

[7] HU, J., GAO, J., POSNER, F.L., et al. Target detection within sea clutter: A comparative study by fractal scaling analyses. Fractals, 2006, vol. 14, no. 3, p. 187-204. DOI: $10.1142 / \mathrm{s} 0218348 \times 06003210$

[8] THAYAPARAN, T., KENNEDY, S. Detection of a manoeuvring air target in sea-clutter using joint time-frequency analysis techniques. IEE Proceedings-Radar, Sonar and Navigation, 2004, vol. 151, no. 1, p. 19-30. DOI: 10.1049/ip-rsn:20040158

[9] DAVIDSON, G., GRIFFITHS, H.D. Wavelet detection scheme for small targets in sea clutter. Electronics Letters, 2002, vol. 38, no. 19, p. 1128-1130. DOI: 10.1049/el:20020790

[10] SEN, S., NEHORAI, A. Target detection in clutter using adaptive OFDM radar. IEEE Signal Processing Letters, 2009, vol. 16, no. 7, p. 592-595. DOI: 10.1109/LSP.2009.2020470

[11] PRASAD, N. N. S. S. R. K., SHAMEEM, V., DESAI, U. B., et al. Improvement in target detection performance of pulse coded Doppler radar based on multicarrier modulation with fast Fourier transform (FFT). IEE Proceedings-Radar, Sonar and Navigation, 2004, vol. 151, no. 1, p. 11-17. DOI: 10.1049/ip-rsn:20040119

[12] SINGH, U. K., MITRA, R., BHATIA, V., et al. Target range estimation in OFDM radar system via kernel least mean square technique. In Proceedings of the IET International Conference on Radar Systems (RADAR). Belfast (UK), 2017, p. 1-5. DOI: 10.1049/cp.2017.0409

[13] SINGH, U. K., MITRA, R., BHATIA, V., et al. Delay and Doppler shift estimation for non-constant envelope modulation in OFDM radar system. In Proceedings of the IET International Conference on Radar Systems (RADAR). Belfast (UK), 2017, p. 1-5. DOI: $10.1049 / \mathrm{cp} .2017 .0410$

[14] ANDRIC, M., BUJAKOVIC, D., BONDZULIC, B., et al. Analysis of radar Doppler signature from human data. Radioengineering, 2014, vol. 23 , no. 1 , p. 11-19. ISSN: 1805-9600

[15] RAIDA, Z., KOLKA, Z., MARSALEK, R., et al. Communication subsystems for emerging wireless technologies. Radioengineering, 2012, vol. 21, no. 4, p. 1036-1049. ISSN: 1805-9600

[16] SHI, C., WANG, F., SELLATHURAI, M., et al. Power minimizationbased robust OFDM radar waveform design for radar and communication systems in coexistence. IEEE Transactions on Signal Processing, 2017, vol. 66, no. 5, p. 1316-1330. DOI: 10.1109/TSP.2017.2770086

[17] GARMATYUK, D., GIZA, P., CONDICT, N., et al. Randomized OFDM waveforms for simultaneous radar operation and asynchronous covert communications. In Proceedings of the IEEE Radar Conference (RadarConf18). Oklahoma City (USA), 2018, p. 975-980. DOI: 10.1109/RADAR.2018.8378693

[18] DOKHANCHI, S. H., SHANKAR, M. B., STIFTER, T., et al. OFDM-based automotive joint radar-communication system. In Proceedings of the IEEE Radar Conference (RadarConf18). Oklahoma City (USA), 2018, p. 902-907. DOI: 10.1109/RADAR.2018.8378680

[19] CHRONOPOULOS, S. K., VOTIS, C., RAPTIS, V., et al. In depth analysis of noise effects in orthogonal frequency division multiplexing systems, utilising a large number of subcarriers. AIP Conference Proceedings, 2010, vol. 1203, no. 1, p. 967-972. DOI: $10.1063 / 1.3322592$

[20] KOLIOPANOS, C., CHRONOPOUlOS, S. K., TZECHILIDOU, A. M., et al. Simulation, modeling, and performance analysis of IEEE 802.16e OFDMA systems for urban and rural environments. In Proceedings of the 2nd International Conference on Signals, Circuits and Systems (SCS). Monastir (Tunisia), 2008, p. 1-4. DOI: 10.1109/ICSCS.2008.4746930 
[21] CHRONOPOULOS, S. K., CHRISTOFILAKIS, V., TATSIS, G., et al. Preliminary BER study of a TC-OFDM system operating under noisy conditions. Journal of Engineering Science and Technology Review, 2016, vol. 9, no. 4, p. 13-16. DOI: 10.25103/jestr.094.03

[22] TIGREK, R. F., DE HEIJ, W. J., VAN GENDEREN, P. OFDM signals as the radar waveform to solve Doppler ambiguity. IEEE Transactions on Aerospace and Electronic Systems, 2012, vol. 48, no. 1, p. 130-143. DOI:10.1109/taes.2012.6129625

[23] LELLOUCH, G., MISHRA, A., INGGS, M. Impact of the Doppler modulation on the range and Doppler processing in OFDM radar In Proceedings of the IEEE Radar Conference. Cincinnati (USA), 2014, p. 803-808. DOI: 10.1109/RADAR.2014.6875700

[24] BRAUN, M., STURM, C., Jondral, F. K. Maximum likelihood speed and distance estimation for OFDM radar. In Proceedings of the IEEE Radar Conference. Washington (USA), 2010, p. 256-261. DOI: 10.1109/RADAR.2010.5494616

[25] ILVEDSON, C. R. Transfer Function Estimation using Time-Frequency Analysis. Master's thesis, Massachusetts Institute of Technology, 1998. Available at: https://dspace.mit.edu/handle/1721.1/50472

[26] PETROV, N., LE CHEVALIER, F., YAROVOY, A. G. Detection of range migrating targets in compound-Gaussian clutter. IEEE Transactions on Aerospace and Electronic Systems, 2018, vol. 54, no. 1, p. 37-50. DOI: 10.1109/TAES.2017.2731558

[27] KAY, S. M. Fundamentals of Statistical Signal Processing. Upper Saddle River (USA): Prentice Hall PTR, 1993. ISBN: 0133457117

[28] LELLOUCH, G., MISHRA, A. K., INGGS, M. Orthogonal frequency division multiplexing phenomenology: Radar technique combining genetic algorithm-based pulse design and energy detector for target recognition. IET Radar, Sonar and Navigation, 2016, vol. 10, no. 5, p. 912-922. DOI: 10.1049/iet-rsn.2014.0470

[29] WARD, K. D., TOUGH, R. J., WATTS, S. Sea Clutter: Scattering, the K Distribution and Radar Performance. Toronto (Canada): IET, 2006. ISBN: 0863415032

[30] SHI, Y., XIE, X., LI, D. Range distributed floating target detection in sea clutter via feature-based detector. IEEE Geoscience and Remote Sensing Letters, 2016, vol. 13, no. 12, p. 1847-1850. DOI: 10.1109/LGRS.2016.2614750

[31] GINI, F., GRECO, M. V. Suboptimum approach to adaptive coherent radar detection in compound-Gaussian clutter. IEEE Transactions on Aerospace and Electronic Systems, 1999, vol. 35, no. 3, p. 1095-1104. DOI: $10.1109 / 7.784077$
[32] CAO, P., XIA, W., LI, Y. Classification of ground targets based on radar micro-Doppler signatures using deep learning and conventional supervised learning methods. Radioengineering, 2018, vol. 27, no. 3 , p. $835-845$. DOI: $10.13164 /$ re.2018.0835

[33] SHBAT, M. S., TUZLUKOV, V. Evaluation of detection performance under employment of the generalized detector in radar sensor systems. Radioengineering, 2014, vol. 23, no. 1, p. 50-65. ISSN: 1805-9600

[34] GINI, F. Sub-optimum coherent radar detection in a mixture of K-distributed and Gaussian clutter. IEE Proceedings-Radar, Sonar and Navigation, 1997, vol. 144, no. 1, p. 39-48. DOI: 10.1049/ip-rsn:19970967

[35] GINI, F., GRECO, M. Covariance matrix estimation for CFAR detection in correlated heavy tailed clutter. Signal Processing, 2002, vol. 82, no. 12, p. 1847-1859. DOI: $10.1016 / \mathrm{S} 0165-1684(02) 00315-8$

[36] ABRAMOWITZ, M., STEGUN, I. A. Handbook of Mathematical Functions: With Formulas, Graphs, and Mathematical Tables. North Chelmsford (USA): Courier Corporation, 1964. ISBN: 0486612724

\section{About the Authors ...}

Uday Kumar SINGH is the research scholar at IIT Indore. He works in the area of radar signal processing.

Vimal BHATIA is currently working a Professor at IIT Indore. He completed Ph.D. from Institute for Digital Communications at The University of Edinburgh (UoE), UK in 2005. His research interests are in the broader areas of nonGaussian non-parametric signal processing with over 180 peer-reviewed publications and 11-patents. He is a reviewer for IEEE, Elsevier, Wiley, Springer, and IET.

Amit Kumar MISHRA is currently a Professor in the Department of Electrical Engineering at the University of Cape Town. Since his Ph.D. (which he finished in the University of Edinburgh in 2006), Prof. Mishra has been an active researcher in the domain of sensor design, radar, applied machine learning and frugal innovation. He is Senior Member of IEEE and his Google-Scholar based H-index is 16. He has more than 150 peer-reviewed publications and 5 patents. 